University of Wollongong

Research Online

Faculty of Engineering and Information

Faculty of Engineering and Information

Sciences - Papers: Part A

Sciences

$1-1-2013$

\title{
99mTc-DTPA volume of distribution, half-life and glomerular filtration rate in normal adults
}

Jennifer L. Holness

Stellenbosch University

John S. Fleming

University Hospital Southampton

Alessandra Malaroda

University of Wollongong, malaroda@uow.edu.au

James M. Warwick

Stellenbosch University

Follow this and additional works at: https://ro.uow.edu.au/eispapers

Part of the Engineering Commons, and the Science and Technology Studies Commons

Research Online is the open access institutional repository for the University of Wollongong. For further information contact the UOW Library: research-pubs@uow.edu.au 


\title{
99mTc-DTPA volume of distribution, half-life and glomerular filtration rate in normal adults
}

\author{
Abstract \\ Assessment of volume of distribution (VD) and half-life (T1/2) values during glomerular filtration rate \\ (GFR) investigations is a useful quality control check. The aim of this study was to derive reference data \\ for VD and T1/2 and also to provide reference data for GFR fro studies performed using $99 \mathrm{mTc}$ - \\ diethylenetriaminepentaacetic acid.

\section{Keywords} \\ normal, adults, dtpa, 99m, filtration, glomerular, life, half, distribution, rate, volume, tc \\ Disciplines \\ Engineering | Science and Technology Studies

\section{Publication Details} \\ Holness, J. L., Fleming, J. S., Malaroda, A. L. \& Warwick, J. M. (2013). 99mTc-DTPA volume of distribution, \\ half-life and glomerular filtration rate in normal adults. Nuclear Medicine Communications, 34 (10), \\ 1005-1014.
}




\section{${ }^{99 m}$ Tc-DTPA volume of distribution, half-life and GFR in normal adults}

Jennifer L. Holness ${ }^{a}$, John S. Fleming ${ }^{b}$, Alessandra L. Malaroda ${ }^{b, c, d}$, James M. Warwick ${ }^{a}$

${ }^{a}$ Nuclear Medicine, Stellenbosch University and Tygerberg Hospital, Cape Town, South Africa

${ }^{b}$ Imaging Physics Group, Medical Physics Department, University Hospital Southampton NHS Foundation Trust, Southampton, UK

'Discipline of Medical Radiation Sciences, Faculty of Health Science, The University of Sydney, Sydney, Australia

dBrain and Mind Research Institute, Faculty of Health Science, The University of Sydney, Sydney, Australia

Correspondence to: Dr Jennifer Holness, Department of Nuclear Medicine, Tygerberg Hospital, Private Bag X3, Tygerberg, 7505, South Africa. Email: jen@sun.ac.za

Requests for reprints to: Dr Jennifer Holness, Department of Nuclear Medicine, Tygerberg Hospital, Private Bag X3, Tygerberg, 7505, South Africa. Email: jen@sun.ac.za

The data were presented previously at the $10^{\text {th }}$ congress of the WFNMB, Cape Town, South Africa, and were published as an abstract in World J Nucl Med 2010; 9(Suppl 1):S-90. 
Abstract

Background and aim Assessment of volume of distribution $\left(V_{D}\right)$ and half-life $\left(T_{1 / 2}\right)$ values during glomerular filtration rate (GFR) investigations is a useful quality control check. The aim of this study was to derive reference data for $V_{D}$ and $T_{1 / 2}$ and also to provide reference data for GFR from studies performed using ${ }^{99 \mathrm{~m}} \mathrm{Tc}$-diethylenetriaminepentaacetic acid $\left({ }^{99 \mathrm{~m}} \mathrm{Tc}\right.$-DTPA).

Methods This was a retrospective study of 126 healthy potential kidney donors (age range 1859 years). GFR was evaluated from ${ }^{99 m}$ Tc-DTPA plasma clearance using the 2004 British Nuclear Medicine Society guidelines. The association between $V_{D}$ and body surface area (BSA) was assessed. $T_{1 / 2}$ was correlated with age and with GFR. The correlation between BrochnerMortensen-corrected GFR (BM-GFR $\left.R_{\text {Corr }}\right)$ and age was evaluated.

Results Uncorrected $V_{D}(L)$ was $\left(10.1^{*} B S A\right) \pm 40.6 \% \quad(p<0.01)$. Corrected $V_{D}(L)$ was $\left(8.19^{*} \mathrm{BSA}\right) \pm 34.4 \%(\mathrm{p}<0.01)$. In individuals under the age of 40 years mean $T_{1 / 2}$ was $95.0 \mathrm{~min} \pm$ $36.2 \%$. In individuals 40 years and older, $T_{1 / 2}$ increased at a rate of $0.49 \mathrm{~min} /$ year $(p=0.04) . T_{1 / 2}$

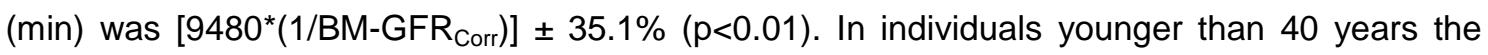
correlation of BM-GFR $\mathrm{Corr}_{\text {and }}$ ane was not statistically significant $(\mathrm{p}=0.45)$ and mean GFR was $108 \mathrm{ml} \cdot \mathrm{min}^{-1} \cdot\left(1.73 \mathrm{~m}^{2}\right)^{-1} \pm 27.5 \%$. In individuals 40 years and older BM-GFR $\mathrm{C}_{\text {corr }}$ was [170 $\left(1.55^{\star}\right.$ age $\left.)\right]\left[\mathrm{ml}^{-m^{-1}} \cdot\left(1.73 \mathrm{~m}^{2}\right)^{-1}\right] \pm 36.7 \%(\mathrm{p}<0.001)$.

Conclusion Well defined reference data for $V_{D}$ and $T_{1 / 2}$ can be used as quality control checks in GFR investigations. In addition to these, reference data for GFR using ${ }^{99 m}$ Tc-DTPA have been defined. This will enhance the interpretation of adult ${ }^{99 \mathrm{~m}}$ Tc-DTPA GFR measurements.

Keywords: ${ }^{99 \mathrm{~m}}$ Tc-DTPA, volume of distribution, half-life, glomerular filtration rate, reference ranges 


\section{Introduction}

Glomerular filtration rate (GFR) is a standard measure of renal function. Although measuring plasma inulin clearance remains the gold standard for determining GFR, this technique is rarely used because it is time-consuming and difficult to perform [1,2]. An estimate of GFR can be obtained by measuring creatinine clearance; however, this technique is inaccurate, especially in cases of poor renal function [3,4]. Measurement of GFR using Nuclear Medicine techniques is considered a suitable alternative with clearance of ${ }^{51} \mathrm{Cr}$-ethylenediaminetetraacetic acid $\left({ }^{51} \mathrm{Cr}\right.$ EDTA) having been shown to be similar to that of inulin $[5,6]$.

${ }^{99 \mathrm{~m}} \mathrm{Tc}$-diethylenetriaminepentaacetic acid $\left({ }^{99 \mathrm{~m}} \mathrm{Tc}\right.$-DTPA) is considered an acceptable alternative to ${ }^{51} \mathrm{Cr}$-EDTA [1,2]. It has the advantages of being inexpensive, widely available and the radiation dose to the patient is low. It is also suitable for gamma camera imaging, allowing simultaneous acquisition of a renogram for calculation of differential renal function. Clearance of ${ }^{99 \mathrm{~m}}$ Tc-DTPA has been shown to correlate well with ${ }^{51} \mathrm{Cr}$-EDTA clearance [7].

In 2004 the British Nuclear Medicine Society (BNMS) published guidelines for the measurement of GFR [2]. The authors recommended measuring the plasma clearance of either ${ }^{51} \mathrm{Cr}$-EDTA or ${ }^{99 \mathrm{~m}}$ Tc-DTPA using the slope-intercept method with Brochner-Mortensen correction [2,8]. In the clinical context this method provides a good compromise between accuracy and simplicity. Nevertheless, careful attention to technique is warranted since methodological errors can be introduced at a number of stages [9]. These include, amongst others, errors in height or weight measurement, drawing up and injection of the patient dose, preparation or measurement of the standard, and preparation or measurement of the plasma samples.

The slope-intercept method does, however, offer a number of opportunities for quality control of the procedure [2]. Two parameters obtained during the calculation of GFR using the slopeintercept method are the volume of distribution $\left(V_{D}\right)$ and the half-life $\left(T_{1 / 2}\right)$ of the injected radiopharmaceutical [8]. While being of limited value for predicting the GFR in isolation, these values can be valuable to check for underlying methodological errors $[2,10,11]$. Using $V_{D}$ and $T_{1 / 2}$ for this purpose requires clearly defined reference ranges for each of these parameters. The BNMS guidelines provide a reference range for the uncorrected $V_{D}(L)$ as being linearly related to body surface area (BSA) $\left(\mathrm{m}^{2}\right)$ by the equation [2]:

$V_{D \text { (uncorrected) }}=\left(8^{*} \mathrm{BSA}\right) \pm 25 \%(2 \mathrm{SD})$

This range for $V_{D}$ was obtained using ${ }^{51} \mathrm{Cr}$-EDTA. It applies to an uncorrected value for $V_{D}$, calculated using the formula: 
$\mathrm{V}_{\mathrm{D} \text { (uncorrected) }}=\mathrm{A} / \mathrm{C}$

where $A$ is the administered activity and $C$ the intercept at zero time obtained by back extrapolation of the terminal exponential of the curve of activity per unit volume versus time [12, Personal communication: G. Blake, King's College London, UK].

The Medical Physics Department of University Hospital Southampton NHS Foundation Trust, UK, found the corrected $V_{D}$ for ${ }^{99 m}$ Tc-DTPA to be related to BSA by the equation [13]:

$V_{D \text { (corrected) }}=\left(6.61^{*} B_{S A} A^{1.218}\right) \pm 32 \%(2 S D)$

The values for $V_{D}$ were calculated using the equation:

$\mathrm{V}_{\mathrm{D} \text { (corrected) }}=\mathrm{BM}-\mathrm{GFR} / \mathrm{k}$

where BM-GFR is the Brochner-Mortensen-corrected GFR [8] and $\mathrm{k}$ is the slope of the terminal exponential.

Equation 2 leads to an overestimation of the volume of distribution as it takes into account only the terminal exponential of the plasma clearance curve after mixing has taken place between the vascular and extravascular compartments [12]. The degree of overestimation is similar to that found when calculating GFR by the slope-intercept method without Brochner-Mortensen correction. The corrected volume of distribution, $V_{D}$ (corrected) (equation 4), although still an approximation since it assumes $\mathrm{k}$ is the terminal exponential, tries to correct for the overestimation.

A technique of measuring extracellular fluid volume (ECV) using a combination of the slope-only and slope intercept methods has been described and validated [14,15]. Using this technique, reference data for ECV have recently been described by Peters et al in a large multi-centre study in the UK [16].

In the BNMS guidelines $T_{1 / 2}$ is described as being "typically in the range" of 100-120 min in adults [2]. However, to the authors' knowledge no data has been published supporting the use of the above or other reference ranges for $V_{D}$ and $T_{1 / 2}$. Specifically, there is a lack of published data for normal values of $V_{D}$ and $T_{1 / 2}$ that have been determined using ${ }^{99 m}$ TC-DTPA [17].

Reference data for GFR have been well-defined by Granerus [18], Hamilton et al [19], Grewal and Blake [20], as well as by Peters et al [16]. Although previous studies have shown only a 
small difference in GFR values obtained using ${ }^{51} \mathrm{Cr}-\mathrm{EDTA}$ and ${ }^{99 \mathrm{~m}} \mathrm{Tc}-\mathrm{DTPA}[21,22]$, there are no published reference ranges for GFR using ${ }^{99 \mathrm{~m}}$ Tc-DTPA.

The aim of this study was to determine reference values for $V_{D}$ and $T_{1 / 2}$ from GFR studies using

${ }^{99 \mathrm{~m}}$ Tc-DTPA in a healthy population. In addition, reference data for GFR using ${ }^{99 \mathrm{~m}}$ Tc-DTPA have been defined for the study population.

\section{Methods}

Patient population:

This retrospective study included the GFR studies of all potential kidney donors referred to the Nuclear Medicine Department of Tygerberg Hospital, Cape Town, South Africa, between February 2007 and September 2012. In total 128 GFR studies were performed and 126 of these were included in the study (69 females, 57 males; age range 18-59 years). Two studies were excluded; one due to discrepancies with weight measurements and one as it was performed using ${ }^{51} \mathrm{Cr}$-EDTA. In 113 subjects a renogram was performed in combination with the GFR study, while in 13 subjects the GFR study was performed on a different day to the renogram. All potential donors underwent a screening process by the hospital's Renal Unit. Subjects were excluded if they had chronic diseases that could potentially affect renal function or that placed them in a high-risk surgical category. Hypertension, diabetes mellitus and psychiatric illness were considered absolute contraindications to kidney donation. The initial blood tests included haematological and biochemical parameters (urea, creatinine, full blood count, liver function, sodium, potassium, chloride, calcium, magnesium, inorganic phosphate, uric acid, glucose), as well as serology for HIV, syphilis, hepatitis $A$, hepatitis $B$, hepatitis $C$, and cytomegalovirus. If these tests were normal and the subject was considered a match based on $A B O$ compatibility and T-cell cross-matching, more specific renal screening was performed. GFR was estimated from a plasma creatinine sample using either the Modification of Diet in Renal Disease (MDRD) [23] or Cockgroft-Gault formula [24]. In addition, creatinine clearance was calculated from a 24hour urine collection, 24-hour urinary protein excretion was determined, and a spot urine sample was collected to determine the protein-to-creatinine ratio. Only if the results of all tests were normal were subjects referred to the Nuclear Medicine department for a renogram and GFR study.

This work was approved by the Stellenbosch University Health Research Ethics Committee; study number N10/05/177.

\section{Measurement of GFR, $\mathrm{V}_{\mathrm{D}}$ and $\mathrm{T}_{1 / 2}$ :}

All GFR studies were performed based on the protocol described in the BNMS guidelines [2]. The subjects' heights and weights were recorded and the BSA calculated using the Haycock 
formula [25]. ${ }^{99 m}$ Tc-DTPA (TechneScan® DTPA, Covidien) was injected intravenously. Labelling efficiency was greater than $90 \%$ in all cases. The injection site was imaged to exclude extravasation. The dose was approximately $40 \mathrm{MBq}$ when only the GFR study was performed on that visit, and about $400 \mathrm{MBq}$ when the GFR study was combined with a renogram. The patient and standard doses were accurately calibrated by weighing the syringes pre- and postinjection on a Precisa $620 \mathrm{C}$ balance, without flushing the syringes or removing the needles. Three $8 \mathrm{ml}$ venous blood samples were drawn from the contralateral arm at 2, 3 and 4 hours respectively. The exact time of injection and the time of drawing each sample were recorded to the nearest minute. Samples were centrifuged immediately after being drawn. A standard was prepared by withdrawing a similar dose of ${ }^{99 m}$ Tc-DTPA from the same kit and adding it to a halffilled $100 \mathrm{ml}$ flask, which was subsequently filled to the $100 \mathrm{ml}$ mark with distilled water and mixed. Two millilitres of this solution was pipetted into a second $100 \mathrm{ml}$ flask that was filled and mixed in a similar manner. The dilution volume of the standard was thus equivalent to 5 litres. Duplicate $1 \mathrm{ml}$ aliquots of plasma samples and standard were pipetted into counting tubes. Background counts were recorded, followed by the counting of each sample in a Picker $\mathrm{Nal}(\mathrm{TI})$ well counter. All samples were counted sequentially in one sitting. Linearity of the well counter was checked routinely and was acceptable, specifically at high count rates.

The GFR was calculated using the slope-intercept method as described in the 2004 BNMS guidelines [2]. The natural logarithm of the plasma ${ }^{99 m}$ Tc-DTPA concentrations were plotted against time. Linear regression analysis was used to determine the half-life $\left(T_{1 / 2}\right)$ and $V_{D}$ (uncorrected). The slope-intercept GFR (SI-GFR) was calculated using the equation [2]:

SI-GFR $=V_{D}$ (Uncorrected) $\times\left(0.693 / T_{1 / 2}\right)$

The SI-GFR was then corrected for body surface area:

SI-GFR Corr $=$ SI-GFR $\times\left(1.73 / \mathrm{BSA} \mathrm{m}^{2}\right)$

Subsequently, the mean Brochner-Mortensen (BM) equation was applied to correct for the missing area under the curve from the fast exponential [2]:

${\mathrm{BM}-\mathrm{GFR}_{\text {Corr }}=1.0004 \times \text { SI-GFR }}_{\text {Corr }}-0.00146 \times \mathrm{SI}^{-\mathrm{GFR}^{2}}{ }_{\text {Corr }}$

The coefficients used in this equation are an average of those in the adult [8] and paediatric equations [26].

The absolute GFR was calculated by reversing the BSA correction: 
BM-GFR = BM-GFR Corr $\times\left(B S A m^{2} / 1.73\right)$

For each GFR study the uncorrected $V_{D}$ was calculated using equation 2 and the corrected $V_{D}$ was calculated using equation 4.

Using the methodology previously described [14-16], ECV-BSA (extracellular volume corrected to a BSA of $1.73 \mathrm{~m}^{2}$ ) was calculated for each individual. Correction for BSA was reversed by multiplication of ECV-BSA with BSA $/ 1.73 \mathrm{~m}^{2}$ to give ECV [16].

\section{Defining reference ranges:}

Values for $\mathrm{V}_{\mathrm{D}}$, both uncorrected and corrected, were plotted against BSA. Using linear regression analysis the correlation was determined between $V_{D}$ and BSA. Variability was defined by calculating the standard error of the estimate of the regression analysis. This gives the standard deviation of estimating $V_{D}$ from BSA. In this report the $95 \%$ confidence limits, or two standard deviations, are expressed as a percentage relative to the mean $V_{D}$ value. These results were compared to the accepted reference ranges described earlier (equations 1 and 3 ) $[2,13]$ and to the ECV-BSA data described by Peters et al [16].

In order to define reference data for $T_{1 / 2}$, the correlations of $T_{1 / 2}$ and age as well as $T_{1 / 2}$ and 1/BMGFR $_{\text {Corr }}$ were determined. Similarly, the association between BM-GFR $R_{\text {Corr }}$ and age was investigated using linear regression. Variability for these parameters was also described by the relative two standard deviation, expressed as a percentage. The results of the BM-GFR $R_{\text {Corr }}$ vs. age correlation were compared to ${ }^{51} \mathrm{Cr}$-EDTA reference ranges described by Granerus [18], Hamilton [19] and Grewal and Blake [20] and to the mean values for GFR described by Peters et al [16].

\section{Deviations from BNMS guidelines}

The protocol used in this study deviated from the BNMS guidelines in two aspects and steps were taken to assess their impact on the calculated GFR and $V_{D}$. Firstly, in 70 of the 126 studies, low counts were recorded for some of the samples. The BNMS guidelines state that, where practical, a minimum of 10000 counts should be obtained from each sample in order to reduce statistical error [2]. The effect of this factor on the accuracy of the GFR and $V_{D}$ was assessed by introducing simulated random error into the counts that were obtained from all samples in all 126 studies. This was repeated 10 times and GFR and $V_{D}$ were calculated in each instance. From this data, systematic, random and total error was estimated.

A second deviation from the BNMS guidelines was that no correction was performed for decay of ${ }^{99 \mathrm{~m}} \mathrm{Tc}$ during the counting process. In order to quantify the error introduced by not correcting for radioactivity decay, an independent set of 26 GFR studies was evaluated. Counts obtained 
from all samples in these studies were higher than 10000 and the exact time at which each sample was counted was recorded. GFR and $V_{D}$ were then calculated for each study, with and without decay correction. From this set of data the systematic, random and combined errors were computed.

\section{Results}

The scatter graphs of the uncorrected $V_{D}(L)$ and corrected $V_{D}(L)$ as a function of the BSA $\left(m^{2}\right)$ are shown in figures 1 and 2 respectively with trendlines representing $\pm 2 S D$.

The correlation between $V_{D}(L)$, both uncorrected and corrected, and BSA $\left(\mathrm{m}^{2}\right)$ was significant $(p<0.001$ for both correlations). Both were best described using linear functions:

$V_{D(\text { Uncorrected })}=(10.1 * B S A) L \pm 40.6 \%(2 S D)$

(RMSE $=3.70 \mathrm{~L} ; 95 \% \mathrm{Cl}$ for the coefficient: 9.79 to $10.5 \mathrm{~L}$ ).

$V_{D \text { (Corrected) }}=(8.19 * B S A) L \pm 34.4 \%(2 S D)$

$(\mathrm{RMSE}=2.53 \mathrm{~L} ; 95 \% \mathrm{Cl}$ for the coefficient: 7.95 to $8.44 \mathrm{~L}$ ).

In figure 1 the trendlines representing the upper and lower limits of the range described in the BNMS guidelines (equation 1) [2] are displayed. Similarly, the trendlines representing the upper and lower limits of the range described by University Hospital Southampton NHS Foundation Trust, UK [13] are displayed in figure 2. 
Fig. 1

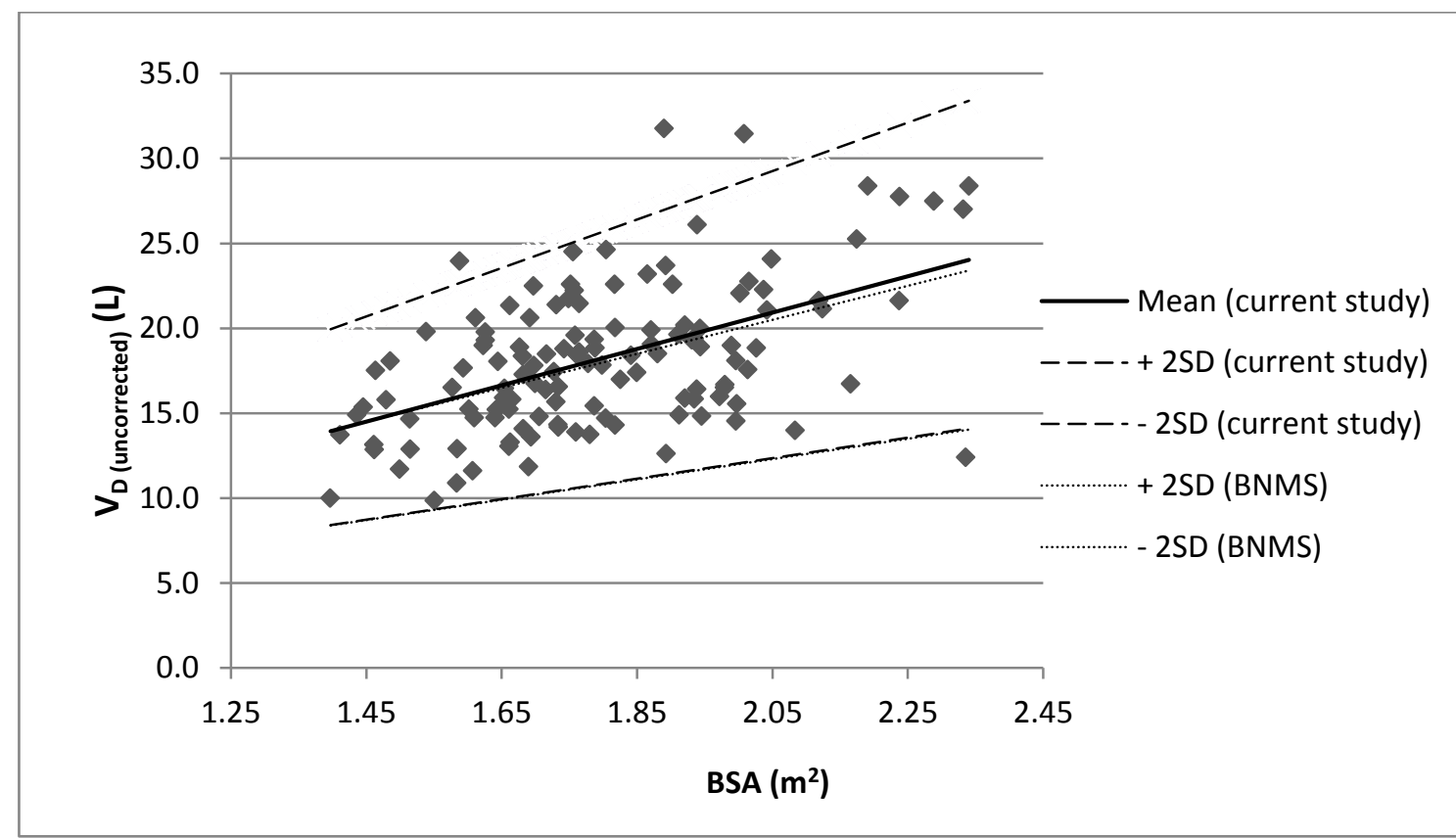

Scatter graph of the uncorrected values of volume of distribution $\left[\mathrm{V}_{\mathrm{D} \text { (uncorrected) }}\right]$ in litres plotted as a function of $\mathrm{BSA}$. The central line represents equation 9, the upper and lower lines (dashes) represent $\pm 2 S D( \pm 40.6 \%)$. The faint dotted lines represent the upper and lower limits of the reference range described in BNMS guidelines (8*BSA $\pm 25 \%$ ) (2SD) [2]. There is overlap of the two lines representing - 2SD.

Fig. 2

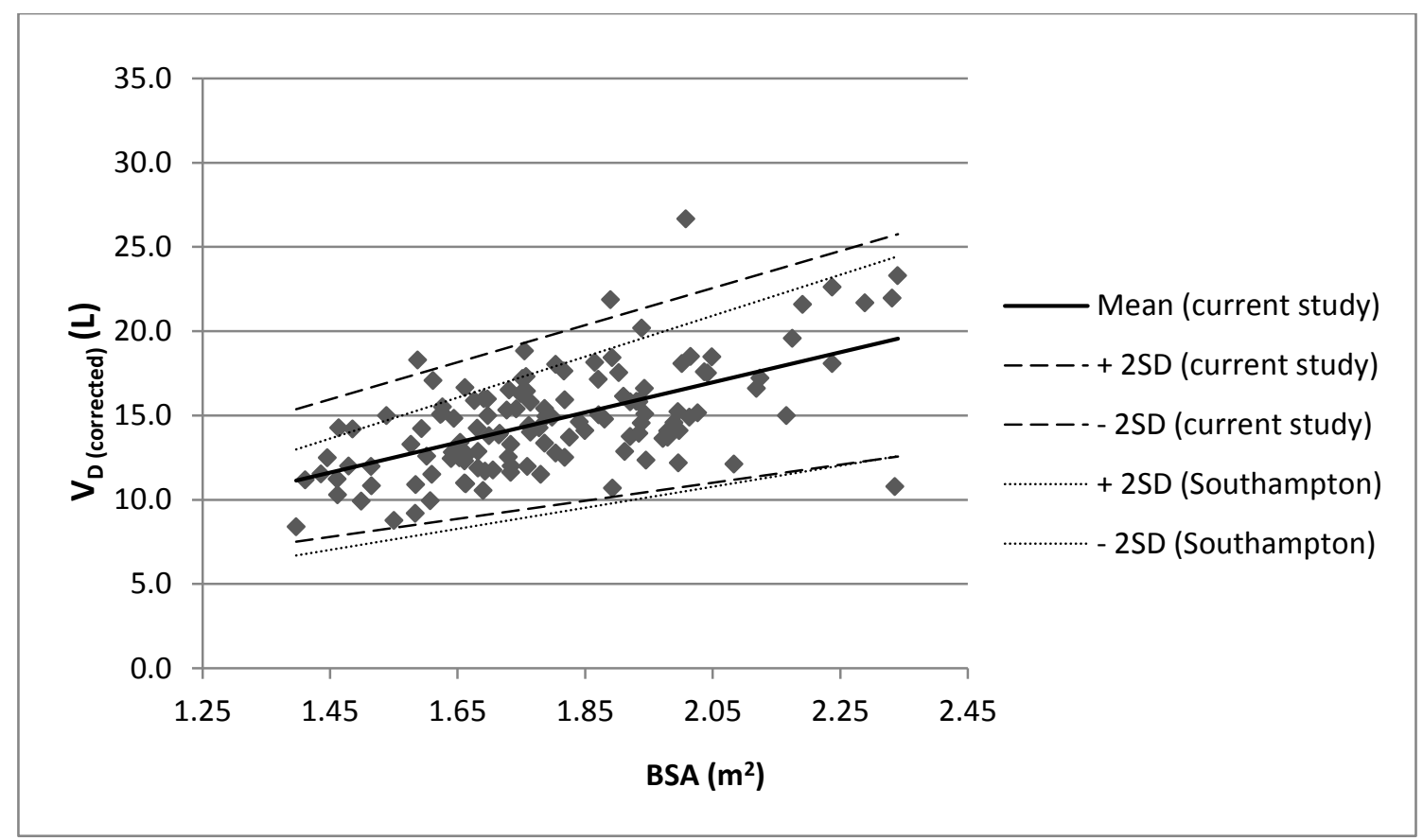

Scatter graph of corrected values of volume of distribution $\left[\mathrm{V}_{\mathrm{D} \text { (corrected) }}\right]$ in litres plotted as a function of BSA. The central line represents equation 10 , the upper and lower lines (dashes) are $\pm 2 S D( \pm 34.4 \%)$. The faint dotted lines represent the upper and lower limits of the reference range determined by University Hospital Southampton $\left(6.61^{*} \mathrm{BSA}^{1.218}\right) \pm 32 \%$ (2SD) [13]. 
Mean ECV normalised for BSA (ECV-BSA) was $12.7 \pm 4.4$ (2SD) L/1.73 $\mathrm{m}^{2}$. ECV-BSA in men was $13.5 \pm 4.9$ (2SD) L/1.73 $\mathrm{m}^{2}$ and in women $12.0 \pm 3.5$ (2SD) L/1.73 $\mathrm{m}^{2}$.

The association between $T_{1 / 2}(\mathrm{~min})$ and age (years) was not statistically significant $(p=0.16)$, nor was the association between $T_{1 / 2}(\mathrm{~min})$ and age (years) in subjects under the age of 40 years $(p$ $=0.65$ ). In this subgroup (< 40 years) the mean $T_{1 / 2}$ was $95.0 \pm 36.2 \%(2 S D)$. In subjects 40 years and older the association between $T_{1 / 2}$ and age was statistically significant $(p=0.046)$. This bi-linear fit is illustrated in figure 3 . Using linear regression the following equation describes the association in subjects 40 years and older:

$\mathrm{T}_{1 / 2}=\left[\left(0.49^{*} \mathrm{age}\right)+75.9\right] \min \pm 30 \%(2 \mathrm{SD})$

The association between $T_{1 / 2}(\min )$ and $1 / B M-G F R_{\text {Corr }}\left(\min .\left(1.73 \mathrm{~m}^{2}\right) \cdot \mathrm{ml}^{-1}\right)$ was statistically significant $(p<0.001)$ and it is illustrated in the scatter graph in figure 4 . Using linear regression it was best described using the equation:

$\mathrm{T}_{1 / 2}=9480^{*}\left(1 / \mathrm{BM}-\mathrm{GFR}_{\text {Corr }}\right) \min \pm 35.1 \%(2 \mathrm{SD})$

Fig. 3

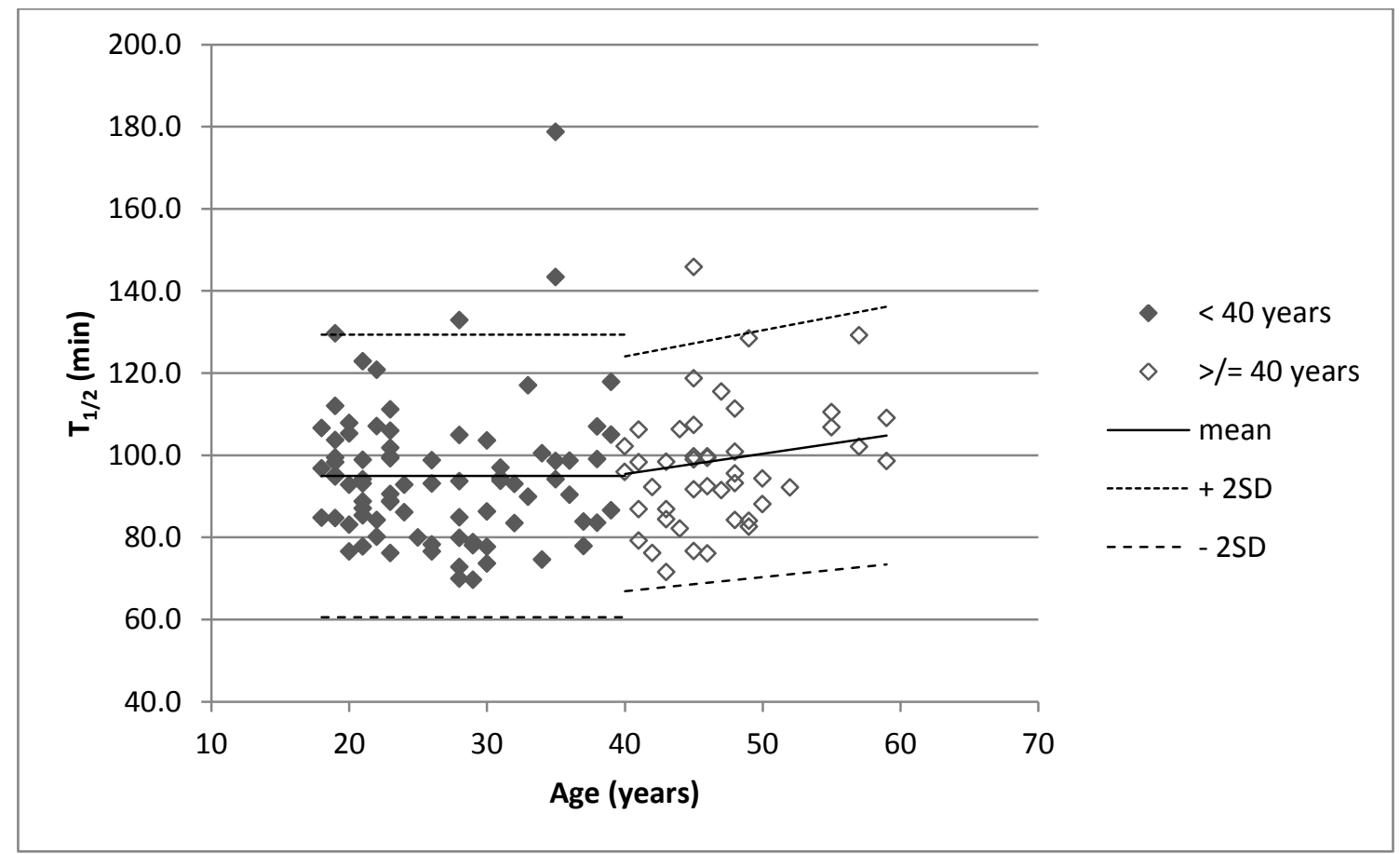

Scatter graph of $T_{1 / 2}(\mathrm{~min})$ plotted as a function of age (years). The central line represents the mean in individuals under the age of 40 years and the equation-predicted-mean in individuals 40 years and older (equation 11). The upper and lower lines are \pm 2 SD $( \pm 36.2 \%$ in individuals $<40$ years and $\pm 36.7 \%$ in individuals $\geq 40$ years 
Fig. 4

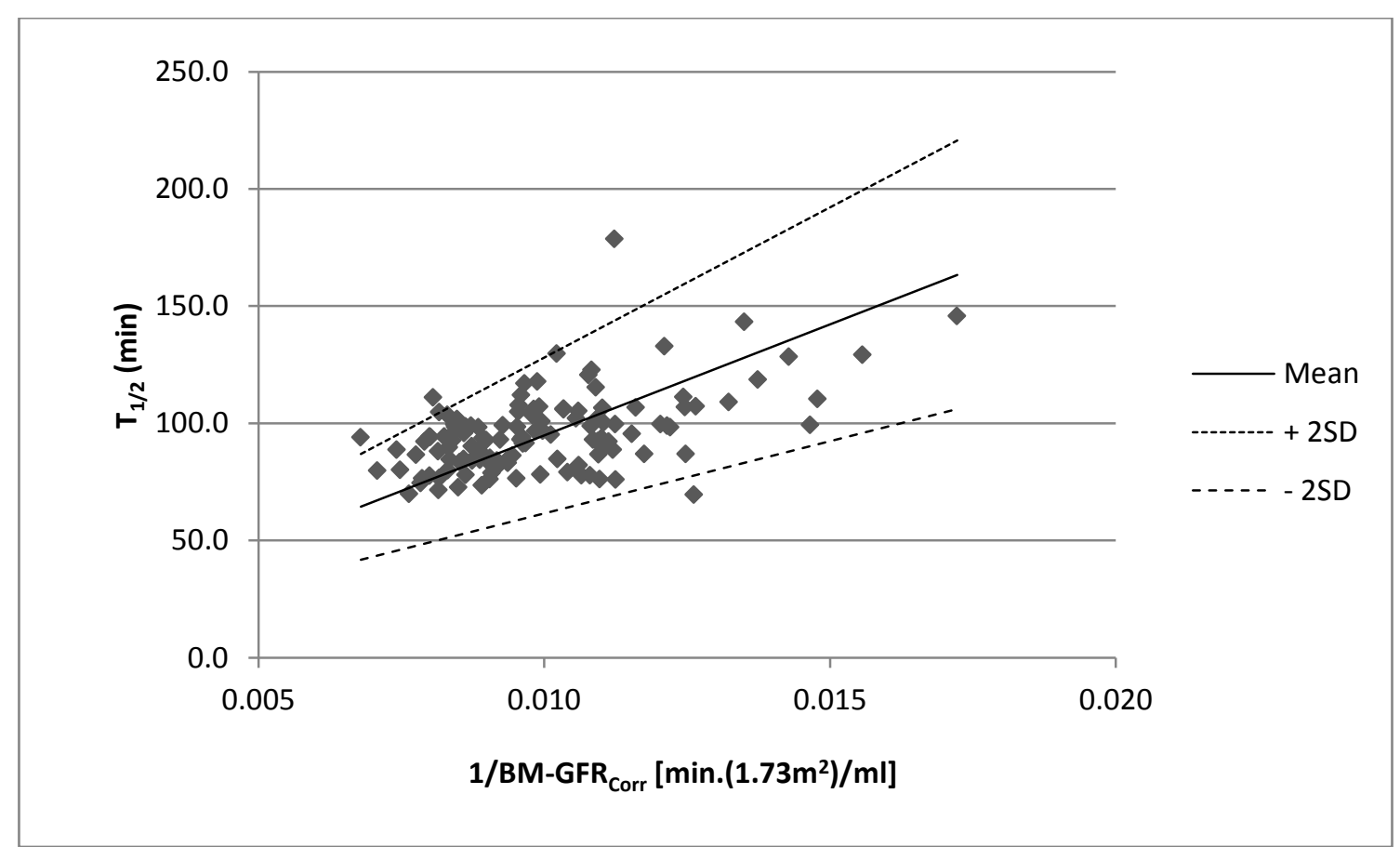

Scatter graph of $T_{1 / 2}(\mathrm{~min})$ plotted as a function of $\left.\left(1 / B M-G F R_{\text {Corr }}\right)\left[\mathrm{min} .\left(1.73 \mathrm{~m}^{2}\right) \cdot \mathrm{ml}^{-1}\right)\right]$. The central line represents $\mathrm{T}_{1 / 2}$ fitted to equation 12 and the upper and lower lines represent $\pm 2 \mathrm{SD}( \pm 35.1 \%)$.

Figure 5 is the scatter graph of BM-GFR $\mathrm{Corr}\left[\mathrm{ml}^{\mathrm{min}} \mathrm{mi}^{-1} \cdot\left(1.73 \mathrm{~m}^{2}\right)^{-1}\right]$ plotted as a function of age (years). In individuals younger than 40 years the correlation was not statistically significant $(p=$ 0.45). The mean GFR in this group was $108 \mathrm{ml}^{-\mathrm{min}^{-1}} \cdot\left(1.73 \mathrm{~m}^{2}\right)^{-1} \pm 27.5 \%$ (2SD). In individuals 40 years and older the correlation between GFR and age was statistically significant $(p<0.001)$. The following equation describes this association:

BM-GFR $\mathrm{Corr}=170-\left(1.55^{\star} \mathrm{age}\right)\left[\mathrm{ml} \cdot \mathrm{min}^{-1} \cdot\left(1.73 \mathrm{~m}^{2}\right)^{-1}\right] \pm 36.7 \%$ (2SD)

Mean BM-GFR $\mathrm{Corr}_{\text {in }}$ men was $107 \pm 29.8$ (2SD) [ml. $\mathrm{min}^{-1} \cdot\left(1.73 \mathrm{~m}^{2}\right)^{-1}$ ] and in women $100.7 \pm$

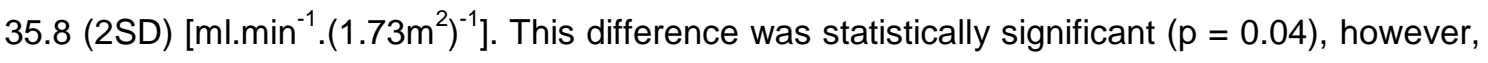
men were significantly younger than women, mean age 30.5 vs. 36.4 years $(p=0.003)$. 
Fig 5

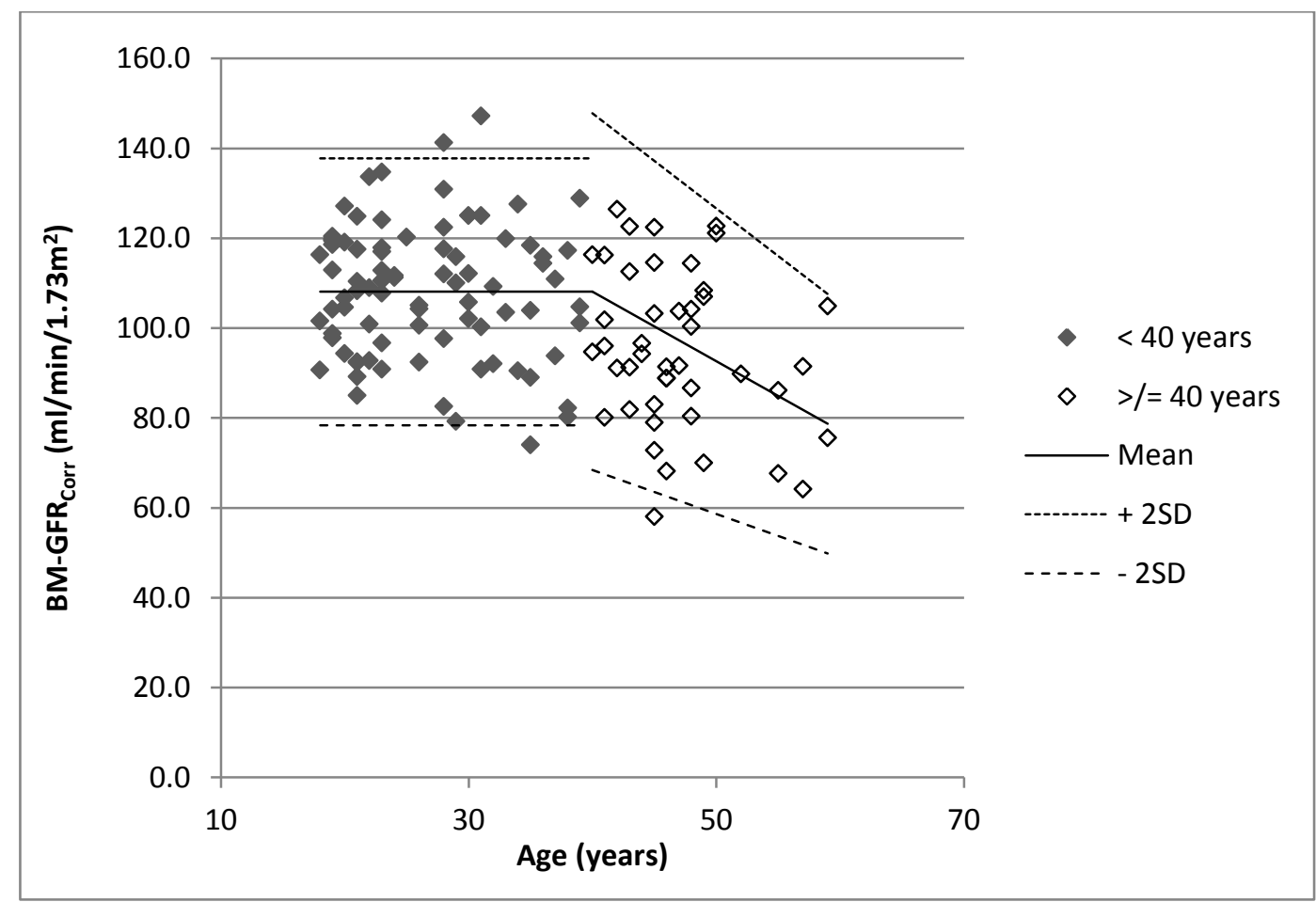

Scatter graph of BM-GFR $\mathrm{Corr}\left[\mathrm{ml}^{\mathrm{min}} \mathrm{m}^{-1} \cdot\left(1.73 \mathrm{~m}^{2}\right)^{-1}\right.$ ] as a function of age (18-59 years) in 126 potential kidney donors. GFR values were corrected for BSA and using the mean Brochner-Mortensen equation [2,8]. The central line represents the mean GFR in individuals under the age of 40 years and the mean fitted to equation 13 in individuals 40 years and older. The upper and lower lines represent \pm 2 SD ( $\pm 27.5 \%$ in individuals under the age of 40 years and $36.7 \%$ in individuals older than 40).

Considering all 126 studies, the systematic and random errors (1SD) introduced to GFR data through statistical noise were $-0.19 \%$ and $2.97 \%$ respectively, and for $V_{D}, 0.64 \%$ and $10.19 \%$ respectively. In the prospective series of 26 studies the systematic and random errors (1SD) introduced to GFR data through not correcting for radioactivity decay were $-0.12 \%$ and $1.81 \%$ respectively, and for $\mathrm{V}_{\mathrm{D}},-0.22 \%$ and $2.37 \%$ respectively. In this series the counting of all samples was completed within 14 minutes (range 6-14 min, mean 9 min).

\section{Discussion}

In this study reference ranges for a South African adult population were determined for $V_{D}, T_{1 / 2}$ and GFR using ${ }^{99 \mathrm{~m}}$ Tc-DTPA and the slope-intercept method as described in the BNMS Guidelines [2]. The slope-intercept method remains prone to methodological errors $[5,6]$ and various quality control checks have been proposed: the fit of the counts to a single exponential can be assessed, either graphically or by checking that the correlation coefficient is greater than 0.985 [2]. Alternatively, slope-intercept GFR measurements can be checked using singlesample estimates $[27,28]$ or using the slope-only technique $[9,14]$. 
$V_{D}$ and $T_{1 / 2}$ are two quantities that are obtained during calculation of GFR using the slopeintercept method. The BNMS guidelines recommend reviewing these quantities as an additional quality control check [2]. For this purpose it is necessary to compare values to normal values for $V_{D}$ and $T_{1 / 2}$ defined for the patient population and for the radiopharmaceutical used.

In the present study a reference range for uncorrected $V_{D}$ in litres was identified as $\left(10.1^{*} B S A\right)$ $\pm 40.6 \%$ (2SD). These values are systematically higher and show greater variability than those described in the BNMS guidelines (equation 1, fig. 1) [2]. Although the values for $V_{D}$ in the BNMS guidelines were derived from GFR measurements using ${ }^{51} \mathrm{Cr}$-EDTA, previous studies demonstrated no significant difference in $V_{D}$ between ${ }^{51}$ Cr-EDTA and ${ }^{99 m}$ TC-DTPA [21, 29]. Therefore, it is believed that it is unlikely that the radiopharmaceutical justifies for the differences between the BNMS range and the values in the current study.

In this study the reference range for corrected $V_{D}$ in litres was found to be $\left(8.19{ }^{*} B S A\right) \pm 34.4 \%$ (2SD). The variability for corrected $V_{D}(34.4 \%)$ is noted to be lower than for uncorrected $V_{D}$ $(40.6 \%)$. This is expected because uncorrected $V_{D}$ is overestimated relative to the true value and the degree of overestimation depends on GFR. Thus a subject of a given size will have a higher value for uncorrected $V_{D}$ if GFR is normal than if it is reduced. This GFR-related variability of $V_{D}$ is reduced by applying a Brochner-Mortensen correction.

In this study the values for corrected $V_{D}$ are systematically higher than those reported by University Hospital Southampton NHS Foundation Trust (equation 3, fig. 2) [13]. For example, for a BSA of $1.73 \mathrm{~m}^{2}$, the corrected $V_{D}$ using the Southampton equation would be $12.9 \mathrm{~L}$ whilst it would be $14.2 \mathrm{~L}$ using equation 11 , leading to a $9 \%$ higher value. However, considering that the 2SD error for the Southampton data is $32 \%$ and for the data in the current study it is $34.4 \%$, the difference in variability between the two centres is within the estimated error on the $V_{D}$.

Radiopharmaceutical factors are even less likely to account for the differences in corrected $V_{D}$ between this centre and Southampton University Hospitals NHS Trust. In fact, both centres used TechneScan $\AA$ DTPA, Covidien. This specifically excludes differences in protein binding of different DTPA preparations as a cause for the higher values or greater variability seen in $V_{D}$.

The study populations in the two centres differ. The Southampton data was obtained from a general clinical GFR population, which included normal and abnormal GFRs and both children and adults, while the data in this study was obtained from a carefully selected normal adult population. This will affect the uncorrected values of $V_{D}$. The overestimation of uncorrected $V_{D}$ will be higher in the normal group compared to the mixed population as the GFR will on average be higher. In terms of environmental and ethnic factors, the population in this study is likely to be more diverse than a population originating from Southampton. The current study population 
is heterogeneous, with roughly equal numbers of subjects of Caucasian, African and mixed ancestry. It has been shown in previous studies that there are differences in muscle mass amongst different ethnic groups and this may translate to differences in $V_{D}$ [30-32].

An additional factor contributing to the variability in the results for $V_{D}$ might have been experimental error due to low counts; however, this is thought to play a minor role and it will be discussed later in this section.

Using the technique described by Peters et al ECV-BSA was calculated for each subject [1416]. The mean ECV-BSA was $12.7 \pm 4.4$ (2SD) L/1.73 $\mathrm{m}^{2}$, whilst for males it was $13.5 \pm 4.9$ (2SD) $\mathrm{L} / 1.73 \mathrm{~m}^{2}$ and females $12.0 \pm 3.5$ (2SD) $\mathrm{L} / 1.73 \mathrm{~m}^{2}$. These GFR values were corrected for the one-pool assumption using the mean Brochner-Mortensen correction as recommended in the BNMS guidelines [2]. When corrected using the adult Brochner-Mortensen equation [8], ECV/BSA in males was $13.9 \pm 5.1$ (2SD) L/1.73 $\mathrm{m}^{2}$ and in females $12.3 \pm 3.7$ (2SD) L/1.73 $\mathrm{m}^{2}$. These values for ECV-BSA agree reasonably well with those described by Peters et al in their recent multi-centre UK-based study [16].

The mean value for ECV in this study is $27 \%$ lower than the mean value for uncorrected $V_{D}$. This is in close agreement with previous work in which a difference of $30 \%$ was described [33]. The mean value for ECV-BSA in the current study is, however, also approximately $10 \%$ lower than the mean value for corrected $V_{D}$ normalized for BSA. This is due to the approximation used in this study that the slope of the second exponential is equal to the clearance constant. The work of Bird et al [15] shows that the slope systematically underestimates the constant by about $10 \%$ leading to an overestimate in the volume of distribution.

Calculation of $T_{1 / 2}$ may be used as a quality control check by comparing it against the value expected for the subject's GFR. The association between $T_{1 / 2}(\mathrm{~min})$ and $1 / B M-G F R_{\text {corr }}$ [min. $\left(1.73 \mathrm{~m}^{2}\right) \cdot \mathrm{ml}^{-1}$ ] was statistically significant $(\mathrm{p}<0.001)$ and $\mathrm{T}_{1 / 2}(\mathrm{~min})$ was found to be $\left[9480^{*}\left(1 /\right.\right.$ BM $^{-G F R}$ Corr $\left.)\right] \pm 35.1 \%$.

In the study by Grewal and Blake, the authors noted that it was apparent that there was a break in the age dependence of GFR at approximately 40 years [20]. They found no statistically significant correlation between GFR and age for individuals under the age of 40 years, while there was a statistically significant decrease in GFR from the age of 40 years onward. In this study a cut-off of 40-years was used based on this work and it supports that conclusion: for individuals under the age of 40 , the correlation between GFR and age was not statistically significant $(p=0.45)$, while it was significant $(p<0.001)$ in individuals 40 years and older. In

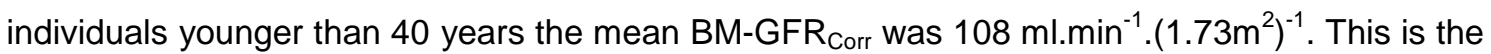
same as the $108 \mathrm{ml} \cdot \mathrm{min}^{-1} \cdot\left(1.73 \mathrm{~m}^{2}\right)^{-1}$ reported by Hamilton et al [19], but higher than the 103 
$\mathrm{ml} \cdot \mathrm{min}^{-1} \cdot\left(1.73 \mathrm{~m}^{2}\right)^{-1}$ reported by Grewal and Blake [20] and the $105 \mathrm{ml}^{-\mathrm{min}^{-1}} \cdot\left(1.73 \mathrm{~m}^{2}\right)^{-1}$ reported by Granerus and Aurell [18]. The slightly higher GFR is expected for DTPA compared to EDTA [21-22]. In individuals 40 years and older, BM-GFR Corr was expressed by the linear relation 170 - $\left(1.55^{\star}\right.$ age $)\left[\mathrm{ml}^{\prime} \mathrm{min}^{-1} \cdot\left(1.73 \mathrm{~m}^{2}\right)^{-1}\right] \pm 36.7 \%$ (2SD). In this study the reference curve predicts a mean GFR at age 50 years of $93 \mathrm{ml} \cdot \mathrm{min}^{-1} \cdot\left(1.73 \mathrm{~m}^{2}\right)^{-1}$. This is in good agreement with the mean of $94 \mathrm{ml} \cdot \mathrm{min}^{-1} \cdot\left(1.73 \mathrm{~m}^{2}\right)^{-1}$ found by Grewal and Blake [20], but lower than the $98 \mathrm{ml} \cdot \mathrm{min}^{-}$ ${ }^{1} .\left(1.73 \mathrm{~m}^{2}\right)^{-1}$ in the Granerus and Aurell study [18]. The data in the subgroup 40 years and older has to be interpreted with caution, however, as it comprised only 44 individuals and covered a relatively limited age range compared to the other studies.

The results of Granerus and Aurell [18] and Hamilton et al [19] are not directly comparable due to small differences in methodology. On the other hand, the current study is based on the protocol described in the BNMS guidelines [2], as was the study by Grewal and Blake [20], making it more appropriate for comparison.

It is accepted that GFR declines with age, although a cut-off age for the start of the decline is difficult to establish as recently shown by Peters et al [16]. No clear age cut-off could be identified in the current study, however a threshold of 40 years of age was chosen in accordance with the cut-off age used in a previous study [20]. Due to a relatively small study sample, individuals were not divided into groups based on gender, however, mean BM-GFR $\mathrm{Corr}_{\text {r }}$ was $107.0 \pm 14.9$ (1SD) ml. $\mathrm{min}^{-1} .\left(1.73 \mathrm{~m}^{2}\right)^{-1}$ in men vs. $100.7 \pm 17.9$ (1SD) $\mathrm{ml} \cdot \mathrm{min}^{-1} .\left(1.73 \mathrm{~m}^{2}\right)^{-1}$ in women. The difference was statistically significant $(p=0.04)$, however, the difference might be explained by the fact that the male cohort was significantly younger than the female cohort (mean age 30.5 versus 36.4 years, $p=0.002$ ). These mean values for $G F R$ in men and women are higher than those described in the multi-centre UK study [16], but this can be explained by two factors: firstly, the majority of GFR measurements (1783 of 1878) in the multi-centre study were performed using ${ }^{51} \mathrm{Cr}$-EDTA and secondly, the mean age of subjects in all the individual centres was higher than the mean age of subjects in the current study. Due to the relatively small study population, individuals in the current study could not be sub-divided into groups based on other factors such as obesity as was done in the multi-centre study [16].

In another study by the same authors the coefficient of variation (CV) of ECV-BSA in normal subjects was found to be useful in assessing departmental performance as it reflects the 'technical robustness' with which the department performs the GFR measurements [34]. The authors suggest a range of $10-20 \%$ as acceptable. The CV for ECV-BSA in this study (using the adult Brochner-Mortensen correction equation [8]) was 15\%.

The BNMS guidelines state that, where practical, a minimum of 10000 counts should be obtained for each sample in order to reduce statistical errors [2]. In this study, 70 of the 126 
GFR studies contained samples with fewer than 10000 counts. The systematic error (1SD) in GFR and $V_{D}$ values due to counting error was found to be low $(0.19 \%$ and $0.64 \%$ respectively). As expected, the random error (1SD) was higher (GFR 2.97\% and $V_{D} 10.19 \%$ ). Another deviation from the BNMS guidelines was that no correction was made for the decay of Tc-99m. The resultant systematic error (1SD) was negligible in all cases (GFR $-0.12 \%$ and $V_{D}-0.22 \%$ ) therefore it was ignored in further calculations. The random error (1SD) was larger (GFR 1.81\% and $V_{D} 2.37 \%$ ). By assuming that the error measured in the 26 cases represented the error introduced through not correcting for decay in all 126 studies, the combined random error of noise and lack of decay correction could be calculated. This resulted in $3.5 \%$ for GFR and $10.5 \%$ for $V_{D}(1 S D)$.

The components of the relatively high combined random error in $V_{D}$ were assessed further. In the correlation of uncorrected $V_{D}$ against BSA, a 1SD error expressed as a percentage of the mean $V_{D}$ is $20.3 \%$ (equation 9). Part of this variation will be genuine variability of $V_{D}$ with $B S A$, $\mathrm{y} \%$, and part due to experimental error. The two components add in quadrature:

$20.3^{2}=y^{2}+10.5^{2}$

The real standard deviation variation of uncorrected $V_{D}$ with $B S A, y$, is therefore $17.4 \%$. Similarly, for corrected $V_{D}$, a $1 S D$ error expressed as a percentage of the mean is $17.2 \%$ (equation 10) and the real standard deviation variation with BSA is $13.6 \%$. These results show that, because the error in $V_{D}$ is relatively large, the contribution of low counts and lack of decay correction to this variability is small.

It is worth mentioning that a cohort of 126 studies is relatively small, therefore further larger studies are recommended to better define reference data for GFR using ${ }^{99 \mathrm{~m}}$ Tc-DTPA. Secondly, the age of all individuals fell between 18 and 59 years, with few over the age of 50, as these were the subjects being considered as kidney donors. Ideally, a study of this nature should include subjects over a wider range of ages, including individuals over the age of 60 years as these are often the patients referred for GFR studies. Moreover, although the hospital's screening process for kidney donors is intensive, it may not have been rigorous enough to exclude all subjects with mild renal pathology.

Having defined the variation of volume of distribution with body surface area and its expected variation, this data may be used for quality control. Studies in which the value of $V_{D}$ lies outside the expected limits for the subject's BSA may be deemed fail the quality control $(Q C)$ test. Considering the corrected $V_{D}$ data shown in Figure 2, two of the studies lie well away from the 2SD limits and therefore may be considered to fail the QC requirements. Using 2SD limits, 5\% of the studies will lie outside the limits due to natural statistical variation, therefore in practical 
use wider limits might be used e.g. 2.5 or 3 SD. Several different methods of calculating volume of distribution exist and it is therefore important that in using this parameter in quality control values must be compared to the corresponding normal range for that particular estimation of the volume. A similar test may be applied to the measured $T_{1 / 2}$. This is compared to the expected limits of $T_{1 / 2}$ for the subject's normalized GFR and if it lies outside these, then the study is deemed to fail the QC test (figure 4). One limitation of the current data in this respect is that it only contains data from control subjects. To obtain a better fit for low GFR further data is required.

\section{Conclusion}

This study has defined reference data for GFR, $V_{D}$ and $T_{1 / 2}$ from GFR studies using ${ }^{99 m}$ TC-DTPA in a healthy South African adult population. $V_{D}$ and $T_{1 / 2}$ values can provide useful quality control checks for GFR studies performed using the slope-intercept method as described in the BNMS guidelines [2]. Reference data for GFR will enhance the interpretation of adult ${ }^{99 m}$ Tc-DTPA GFR measurements. The small difference in normal values for GFR in comparison to previous studies using ${ }^{51} \mathrm{Cr}$-EDTA is in agreement with previous publications.

\section{Acknowledgements}

Justin Harvey

(Centre for Statistical Consultation, Stellenbosch University) 


\section{References}

1. Blaufox MD, Aurell M, Bubeck B, Fommei E, Piepsz A, Russell C et al. Report of the Radionuclides in Nephrourology Committee on renal clearance. J Nucl Med 1996; 37:1883-1890.

2. Fleming JS, Zivanovic MA, Blake GM, Burniston M, Cosgriff PS. Guidelines for the measurement of glomerular filtration rate using plasma sampling. Nucl Med Commun 2004; 25:759-769.

3. Brochner-Mortensen J, Rodbro P. Selection of routine method for determination of glomerular filtration rate in adult patients. Scand J Clin Lab Invest 1976; 36:35-43.

4. Sawyer WT, Canaday BR, Poe TE, A multicenter evaluation of variables affecting the predictability of creatinine clearance. Am J Clin Pathol 1982; 78:832-838.

5. Garnett ES, Parsons V, Veall N. Measurement of glomerular filtration rate in man using ${ }^{51} \mathrm{Cr} /$ Edetic-acid-complex. Lancet 1967; 1:818-819.

6. Brochner-Mortensen J, Giese J, Rossing N. Renal inulin clearance versus total plasma clearance of Cr-51 EDTA. Scand J Clin Lab Invest 1969; 23:301-305.

7. Hilson AJW, Mistry RD, Maisey MN. Technetium-99m-DTPA for the measurement of glomerular filtration rate. Br J Radiol 1976; 49:794-796.

8. Brochner-Mortensen J. A simple method for the determination of glomerular filtration rate. Scand J Clin Lab Invest 1972; 30:271-274.

9. Bird NJ, Peters C, Michell AR, Peters AM. Comparison between slope-intercept and slope-only techniques for measuring glomerular filtration rate: Use of two independent markers and an independent arbiter. Nucl Med Commun 2007; 28:711-718.

10. Piepsz A, Colarinha P, Gordon I, Hahn K, Olivier P, Sixt R et al. Guidelines for glomerular filtration rate determination in children. Eur J Nucl Med 2001; 28:BP31-6.

11. Piepsz A, Ham R, De Sadeleer C. Correspondence. Nucl Med Commun 2005; 26:175178.

12. Waller DG, Keast CM, Fleming JS, Ackery DM. Measurement of glomerular filtration rate with Technetium-99m DTPA: Comparison of plasma clearance techniques. J Nucl Med 1987; 28:372-377.

13. Fleming JS, Persaud L, Hunt J. Quality control of GFR measurements using volume of distribution. Nucl Med Commun 2009; 30:371.

14. Peters AM. Expressing glomerular filtration rate in terms of extracellular fluid volume. Nephrol Dial Transplant 1992; 7:205-210.

15. Bird NJ, Michell AR, Peters AM. Accurate measurement of extracellular fluid volume from the slope/intercept technique after bolus injection of a filtration marker. Physiol Meas 2009; 30:1371-1379.

16. Peters AM, Perry L, Hooker CA, Howard B, Neilly MDJ, Seshadri N et al. Extracellular fluid volume and glomerular filtration rate in 1878 healthy potential renal transplant 
donors: effects of age, gender, obesity and scaling. Nephrol Dial Transplant 2012; 27:1429-1437.

17. Fleming JS, Zivanovic MA, Blake GM, Burniston M, Cosgriff PS. Reply to Controversies in the estimation of glomerular filtration rate. Nucl Med Commun 2006; 27:407-408.

18. Granerus G, Aurell M. Reference values for ${ }^{51} \mathrm{Cr}$-EDTA clearance as a measure of glomerular filtration rate. Scand J Clin Lab Invest 1981; 41:611-616.

19. Hamilton D, Riley P, Miola U, Mousa D, Popovich W, Al Khader A. Total plasma clearance of ${ }^{51} \mathrm{Cr}$-EDTA: Variation with age and sex in normal adults. Nucl Ned Commun 2000; 21: 187-192.

20. Grewal GS, Blake GM. Reference data for ${ }^{51} \mathrm{Cr}$-EDTA measurements of the glomerular filtration rate derived from live kidney donors. Nucl Med Commun 2005; 26:61-65.

21. Fleming JS, Wilkinson J, Oliver RM, Ackery DM, Blake GM, Waller DG. Comparison of radionuclide estimation of glomerular filtration rate using technetium $99 \mathrm{~m}$ diethylenetriaminepentaacetic acid and chromium 51 ethylenediaminetetraacetic acid. Eur J Nucl Med 1991; 18:391-395.

22. Biggi A, Viglietti A, Farinelli MC, Bonada C, Camuzzini G. Estimation of glomerular filtration rate using chromium-51 ethylene diamine tetra-acetic acid and technetium99m diethylene triamine penta-acetic acid. Eur J Nucl Med 1995; 22:532-536.

23. Levey AS, Bosch JP, Lewis JB, Greene T, Rogers N, Roth D. A more accurate method to estimate glomerular filtration rate from serum creatinine: a new prediction equation. Modification of Diet in Renal Disease Study Group. Ann Intern Med 1999; 130:461-470.

24. Cockgroft DW, Gault MH. Prediction of creatinine clearance from serum creatinine. Nephron 1976; 16:31-41.

25. Haycock GB, Schwarz GJ. Geometric method for measuring body surface area: A height-weight formula validated in infants, children, and adults. $J$ Pediat 1978; 93:6268.

26. Brochner-Mortensen J, Haahr J, Christoffersen J. A simple method for accurate assessment of the glomerular filtration rate in children. Scand J Clin Lab Invest 1974; 33:139-143.

27. Fleming JS, Persaud L, Zivanovic MA. A general equation for estimating glomerular filtration rate from a single plasma sample. Nucl Med Commun 2005; 26:743-748.

28. Fleming JS, Persaud L, Zivanovic MA. Quality control of slope-intercept measurements of glomerular filtration rate using single-sample estimates. Nucl Med Commun 2005; 26:737-742.

29. Rehling M, Møller ML, Thamdrup B, Lund JO, Trap-Jensen J. Simultaneous measurement of renal clearance and plasma clearance of ${ }^{99 \mathrm{~m}} \mathrm{Tc}$-labelled diethylenetriaminepenta-acetate, ${ }^{51} \mathrm{Cr}$-labelled ethylenediaminetetra-acetate and inulin in man. Clin Sci 1984; 66:613-619. 
30. Cohn SH, Abesamis C, Zanzi I, Aloia JF, Yasumura S, Ellis KJ. Body elemental composition: comparison between black and white adults. Am J Physiol 1977; 232:E419-422.

31. Harsha DW, Frerichs RR, Berenson GS. Densitometry and anthropometry of black and white children. Hum Biol 1978; 50:261-280.

32. Worrall JG, Phongsathorn V, Hooper RJL, Paice EW. Racial variation in serum creatine kinase unrelated to lean body mass. Br J Rheumatol 1990; 29:371-373.

33. Peters AM, Henderson BL, Lui D. Indexed glomerular filtration rate as a function of age and body size. Clin Sci 2000; 98: 439-444.

34. Peters AM, Howard B, Neilly MDJ, Seshadri N, Sobnack R, Hooker CA et al. The reliability of glomerular filtration rate measured from plasma clearance: a multi-centre study of 1878 healthy potential renal transplant donors. Eur J Nucl Med 2012; 39: 715722. 


\section{Legend to figures}

Figure 1

Scatter graph of the uncorrected values of volume of distribution $\left[V_{D \text { (uncorrected) }}\right]$ in litres plotted as a function of BSA. The central line represents equation 9, the upper and lower lines (dashes) represent \pm 2 SD $( \pm 40.6 \%)$. The faint dotted lines represent the upper and lower limits of the reference range described in BNMS guidelines ( $8^{*} \mathrm{BSA} \pm 25 \%$ ) (2SD) [2]. There is overlap of the two lines representing - 2SD.

Figure 2

Scatter graph of corrected values of volume of distribution $\left[V_{D}\right.$ (corrected) $]$ in litres plotted as a function of BSA. The central line represents equation 10, the upper and lower lines (dashes) are \pm 2 SD ( $\pm 34.4 \%)$. The faint dotted lines represent the upper and lower limits of the reference range determined by University Hospital Southampton $\left(6.61^{*} \mathrm{BSA}^{1.218}\right) \pm 32 \%$ (2SD) [13].

Figure 3

Scatter graph of $T_{1 / 2}(\mathrm{~min})$ plotted as a function of age (years). The central line represents the mean in individuals under the age of 40 years and the equation-predicted-mean in individuals 40 years and older (equation 11). The upper and lower lines are \pm 2 SD $( \pm 36.2 \%$ in individuals < 40 years and $\pm 36.7 \%$ in individuals $\geq 40$ years).

Figure 4

Scatter graph of $T_{1 / 2}(\mathrm{~min})$ plotted as a function of $\left.\left(1 / B M-G F R_{\text {Corr }}\right)\left[\min .\left(1.73 \mathrm{~m}^{2}\right) \cdot \mathrm{ml}^{-1}\right)\right]$. The central line represents $T_{1 / 2}$ fitted to equation 12 and the upper and lower lines represent \pm 2SD $( \pm$ $35.1 \%)$.

\section{Figure 5}

Scatter graph of BM-GFR $\mathrm{Corr}\left[\mathrm{ml}^{\mathrm{min}} \mathrm{min}^{-1} \cdot\left(1.73 \mathrm{~m}^{2}\right)^{-1}\right.$ ] as a function of age (18-59 years) in 126 potential kidney donors. GFR values were corrected for BSA and using the mean BrochnerMortensen equation [2,8]. The central line represents the mean GFR in individuals under the age of 40 years and the mean fitted to equation 13 in individuals 40 years and older. The upper and lower lines represent \pm 2 SD ( $\pm 27.5 \%$ in individuals under the age of 40 years and $36.7 \%$ in individuals older than 40 ). 